\title{
Risk factors for the development of acute respiratory distress syndrome in mechanically ventilated adults in Peru: a multicenter observational study
}

Ena Gupta ${ }^{1,2}$, Shakir Hossen ${ }^{1,2}$, Matthew R. Grigsby ${ }^{1,2}$, Phabiola Herrera ${ }^{1,2}$, Rollin Roldan ${ }^{3}$, Enrique Paz ${ }^{4}$ Amador A. Jaymez $z^{5}$, Eduardo E. Chirinos ${ }^{6}$, Jose Portugal ${ }^{3}$, Rocio Quispe ${ }^{3}$, Roy G. Brower ${ }^{1}$, William Checkley ${ }^{1,2^{*}}$ (D) and INTENSIVOS Cohort Study

\begin{abstract}
Background: Clinical and epidemiological differences between acute respiratory distress syndrome (ARDS) that presents at the initiation of mechanical ventilation [MV] (ARDS at MV onset) and that which develops during the course of MV (ARDS after MV onset) are not well understood. We conducted an observational study in five Peruvian ICUs to characterize differences between ARDS at MV onset and after MV onset and identify risk factors for the development of ARDS after MV onset.

Methods: We consecutively enrolled critically ill patients with acute respiratory failure requiring at least $24 \mathrm{~h}$ of mechanical ventilation and followed them prospectively during the first 28 days and compared baseline characteristics and clinical outcomes by ARDS status.

Results: We enrolled 1657 participants on MV (mean age 60.0 years, 55\% males) of whom 334 (20.2\%) had ARDS at MV onset and 180 (10.9\%) developed ARDS after MV onset. Average tidal volume at the initiation of MV was 8.7 $\mathrm{mL} / \mathrm{kg}$ of predicted body weight (PBW) for participants with ARDS at MV onset, $8.6 \mathrm{~mL} / \mathrm{kg}$ PBW for those who developed ARDS after MV onset, and $8.5 \mathrm{~mL} / \mathrm{kg}$ PBW for those who never developed ARDS ( $p=0.23$ ). Overall, 90day mortality was 56\% and 55\% for ARDS after MV onset and ARDS at MV onset, respectively, as compared to $46 \%$ among those who never developed ARDS $(p<0.01)$. Adults with ARDS had a higher body mass index (BMI) than those without ARDS ( 27.3 vs $26.5 \mathrm{~kg} / \mathrm{m}^{2}, p<0.01$ ). Higher peak pressure (adjusted interquartile $\mathrm{OR}=1.51,95 \% \mathrm{Cl}$ 1.21-1.88), higher mean airway pressure (adjusted interquartile $\mathrm{OR}=1.41,95 \% \mathrm{Cl} 1.13-1.76$ ), and higher positive end-expiratory pressure (adjusted interquartile $\mathrm{OR}=1.29,95 \% \mathrm{Cl} 1.10-1.50$ ) at MV onset were associated with a higher odds of developing ARDS after MV onset.

Conclusions: In this study of mechanically ventilated patients, 31\% of study participants had ARDS at some point during their ICU stay. Optimal lung-protective ventilation was not used in a majority of patients. Patients with ARDS after MV onset had a similar 90-day mortality as those with ARDS at MV onset. Higher airway pressures at MV onset, higher PEEP, and higher BMI were associated with the development of ARDS after MV onset.
\end{abstract}

Keywords: Acute respiratory distress syndrome, Prevention, Critically ill, Mechanical ventilation

\footnotetext{
* Correspondence: wcheckl1@jhmi.edu

'Division of Pulmonary and Critical Care, School of Medicine, Johns Hopkins

University, 1830 E. Monument St, Room 555, Baltimore, MD 21287, USA

${ }^{2}$ Center for Global Non-Communicable Disease Research and Training,

School of Medicine, Johns Hopkins University, Baltimore, USA

Full list of author information is available at the end of the article
}

(c) The Author(s). 2019 Open Access This article is distributed under the terms of the Creative Commons Attribution 4.0 International License (http://creativecommons.org/licenses/by/4.0/), which permits unrestricted use, distribution, and reproduction in any medium, provided you give appropriate credit to the original author(s) and the source, provide a link to the Creative Commons license, and indicate if changes were made. The Creative Commons Public Domain Dedication waiver (http://creativecommons.org/publicdomain/zero/1.0/) applies to the data made available in this article, unless otherwise stated. 


\section{Background}

Acute respiratory distress syndrome (ARDS) is an inflammatory process leading to diffuse edema and lifethreatening hypoxemic respiratory failure, which can result from a variety of insults. It is characterized by increased pulmonary vascular permeability, increased lung weight, and loss of aerated lung [1]. Current estimates of mortality range from 26 to $58 \%$ with that of low- and middle-income countries (LMICs) in the higher end of this range [2-6]. Indeed, a large, prospective multinational observational study found that low per capita gross national income was associated with lower survival from ARDS [7].

Over the last five decades, extensive basic research has elucidated molecular mechanisms of pathogenesis in ARDS and numerous clinical trials have led to better clinical management for this condition [8-15]. Despite these advances, treatment of ARDS remains mainly supportive. A more preventive approach towards early identification and institution of therapies early in the disease process might have higher success in reducing mortality. The majority of evidence for the diagnosis and management ARDS, however, has stemmed from research conducted in high-income countries. There is a clear need for identification of burden of ARDS and associated morbidity and mortality in resource-poor settings of LMICs [16].

The broad phenotype and etiological causes of ARDS raise the possibility that heterogeneity in ICU characteristics is an important factor in explaining clinical outcomes across settings. These include differences in resources available in the ICU such as health care providers [17], equipment [18], and health care dollars spent per capita [19]. Since being on mechanical ventilation is associated with a higher mortality in LMICs [20], a better characterization of ARDS among mechanically ventilated patients in low-resource settings can help guide changes in critical care delivery. For example, a singlecenter study by Li et al. reported that changes in critical care structure and delivery were temporally associated with a decrease in the incidence of hospital-acquired ARDS with no change in incidence of ARDS on presentation over an 8-year period [21]. As such, it is crucial to understand risk factors for nosocomial ARDS as a step towards prevention of this condition.

To address these gaps in knowledge, we conducted a multicenter, prospective, longitudinal study of adult, mechanically ventilated patients in five ICUs in Lima, Peru. We sought to characterize the proportions of mechanically ventilated patients with ARDS at mechanical ventilation onset and those who developed ARDS after mechanical ventilation onset while in the ICU. Consequently, we evaluated for differences in etiology, clinical characteristics, mechanical ventilation management, and associated morbidity and mortality in these two groups of patients with ARDS. We also evaluated risk factors for the development of ARDS among those who did not have ARDS at mechanical ventilation onset. With this information, we aimed to identify a high-risk population, health care practices, or factors that help to identify participants who develop ARDS after mechanical ventilation onset while in the ICU.

\section{Methods \\ Study setting}

This study was conducted in five ICUs at four public hospitals of the Social Security System (ESSALUD) and Ministry of Health (MINSA) in Lima, Peru. Participating ICUs were selected based on high case volume and willingness to participate. All participating ICUs were closed ICUs with a 24-h in-hospital attending intensivist coverage and with a variable number of critical care fellows and residents depending on the size of the teaching program. Reported annual ICU mortality was similar between units, ranging from 16.7 to $22.9 \%$. None of the ICUs have multidisciplinary rounds or respiratory therapists. There are no established ventilation protocols in use at the Peruvian ICUs; ventilator settings are based on intensivist preference. Details of the characteristics of the participating ICUs and their organization and structure are described elsewhere [22]. We received ethics approval and permission to conduct this study in each of the participating institutions: Hospital Nacional Edgardo Rebagliati Martins, Hospital Nacional Guillermo Almenara Irigoyen, Hospital Nacional Arzobispo Loayza, and Hospital de Emergencias Casimiro Ulloa. Ethics approvals were obtained from the institutional review boards of A.B. PRISMA and ESSALUD Hospital Nacional Edgardo Rebagliati Martins in Lima, Peru, and the Johns Hopkins School of Medicine in Baltimore, USA. We obtained a waiver of written informed consent from all institutions to conduct this observational study.

\section{Study design}

INTENSIVOS (critical in Spanish) is a prospective, observational cohort study. Subjects were consecutively enrolled between December 2010 and October 2013 based on the following eligibility criteria: age $\geq 18$ years, at least $24 \mathrm{~h}$ of invasive mechanical ventilation in one of the ICUs participating in the study, and enrollment into the study within $48 \mathrm{~h}$ of onset of mechanical ventilation. We also assessed disease severity using the SOFA (Sepsis-related Organ Failure Assessment), the SAPS II (Simplified Acute Physiology Score), and the APACHE II and APACHE III (Acute Physiology And Chronic Health Evaluation) scores [23-25]. We obtained demographic, chronic disease, and acute physiological data for all participants meeting eligibility criteria. Participants were 
followed daily to monitor vital status, clinical and ventilator management, acute physiology, and use of sedation during their ICU stay for up to 28 days in the ICU, until ICU discharge, or death whichever came first. Participants successfully discharged from the ICU were followed for vital status during their inpatient hospital stay. All participants were contacted at 90 days after enrolment to assess their vital status.

\section{Outcomes}

The primary outcome of this analysis was the development of ARDS after mechanical ventilation onset according to the Berlin definition [17]. Although the study was designed before the Berlin definition was published, we were able to combine individual components of the Berlin definition at the analysis stage. Secondary outcomes for this analysis were 90-day mortality, ventilatorfree days at 28 days, ICU-free days at 28 days, and hospital-free days at 60 days.

\section{Definitions}

We divided study participants into three categories by their ARDS status during the course of the study: no ARDS at any time during the study period, ARDS at mechanical ventilation onset. and ARDS after mechanical ventilation onset as that which develops while on mechanical ventilation during their ICU stay. We further classified ARDS after mechanical ventilation onset as early if it developed in $<7$ days after initiation of mechanical ventilation and late if it developed in $\geq 7$ days after initiation of mechanical ventilation.

\section{Risk factors}

Risk factors were defined a priori based on previously published studies showing associations of risk factors for the development of ARDS and ventilator parameters of interest. These included age, sex, APACHE II, SAPS II and SOFA severity scores, body mass index (BMI) [26], tidal volume [27, 28], inspiratory plateau pressure, peak airway pressure, mean airway pressure, driving pressure, and static respiratory system compliance. Plateau pressure was obtained by performing an end-inspiratory hold maneuver on the ventilator that lasted at least $0.5 \mathrm{~s}$. Driving pressure was measured as the difference between plateau pressure and positive end-expiratory pressure (PEEP). Static compliance was measured as tidal volume divided by driving pressure. Fluid balance in the first $24 \mathrm{~h}$ was calculated as the difference between total intake and urine output over $24 \mathrm{~h}$.

\section{Biostatistical methods}

We first calculated proportions of mechanically ventilated participants with ARDS at mechanical ventilation onset and with ARDS after mechanical ventilation onset in our cohort. We then estimated the incidence of ARDS after mechanical ventilation onset as the number of mechanically ventilated participants who developed ARDS during their ICU stay divided by the number of ICU days among those without ARDS at mechanical ventilation onset (standardized to 100 ICU days), and used standard methods to calculate a $95 \%$ confidence interval.

To visualize the progression of clinical outcomes over time by ARDS status, we graphed the cumulative incidences of death, of achieving unassisted breathing, and of being alive in the hospital and not achieving unassisted breathing. We compared all potential risk factors including ventilator parameters at the initiation of mechanical ventilation and clinical outcomes by ARDS status. We compared groups with chi-square or Fisher exact tests for categorical values, and $t$ tests, Wilcoxon rank sum tests or analysis of variance for continuous variables, as appropriate. We used multivariable logistic regression to compare 90-day mortality by ARDS status and used multivariable linear regression to compare ventilator-, ICU-, and hospital-free days by ARDS status adjusted for age, sex, body mass index, APACHE III, and an indicator variable for ICU.

We then used the cohort of participants who did not have ARDS at mechanical ventilation onset to identify predictors of ARDS after mechanical ventilation onset. We conducted single variable logistic regression to model the odds of developing ARDS after mechanical ventilation onset by age, sex, height, BMI, APACHE II, APACHE III, SOFA, SAPS-2, fraction of inspired oxygen $\left(\mathrm{FiO}_{2}\right)$ at the initiation of mechanical ventilation, tidal volume at the initiation of mechanical ventilation, mean airway pressure at the initiation of mechanical ventilation, peak airway pressure at the initiation of mechanical ventilation, plateau pressure at the initiation of mechanical ventilation, driving pressure at the initiation of mechanical ventilation, static respiratory system compliance at the initiation of mechanical ventilation, PEEP at the initiation of mechanical ventilation, $\mathrm{PaO}_{2} / \mathrm{FiO}_{2}$ at the initiation of mechanical ventilation, the difference between actual and predicted body weight (ABW-PBW), and vasopressor use fluid balance in the first $24 \mathrm{~h}$. Finally, given that airway pressures were associated with the development of ARDS after mechanical ventilation onset, we performed separate multivariable logistic regressions for all airway pressures collected at the initiation of mechanical ventilation including mean airway pressure, peak airway pressure, plateau pressure, driving pressure, PEEP, and static respiratory system compliance adjusted for age, sex, body mass index, APACHE III, and an indicator variable for ICU. We ran these adjusted regression models separately to avoid potential collinearity. We also ran separate models for BMI, the difference 
between actual and predicted body weight, and fluid balance adjusted for age, sex, APACHE III, and an indicator variable for ICU. Finally, we performed subgroup analyses as above to compare baseline characteristics, ventilatory parameters, and clinical outcomes between those who developed early and late ARDS after mechanical ventilation onset.

Analyses were conducted using Stata 12.1 (StatCorp, College Station, Texas) and R (www.r-project.org).

\section{Results}

\section{Participant characteristics}

We screened 1858 participants, of which 116 (6\%) did not meet the criteria for enrollment. Of the 1742 enrolled participants, $83(4.7 \%)$ had missing baseline or daily clinical information and $2(0.1 \%)$ were missing a study termination form. A total of 1657 participants were enrolled and had complete data for analysis (Additional file 1: Fig. S1). Mean \pm SD age was $60.0 \pm 18.9$ years, 907 (54.7\%) were males, $72.3 \%$ were admitted for medical reasons, and the remaining $27.7 \%$ were either admitted for trauma or were post-surgical admissions (Table 1). We also plotted the percentage of ICU days by day of mechanical ventilation for which a chest radiograph or arterial blood gas was obtained after mechanical ventilation onset (Additional file 1: Fig. S2).

\section{Ascertainment of ARDS in the ICU}

Of the 1657 mechanically ventilated participants, 514 (31\%) met criteria for ARDS during the first 28 days, of which $334(20.2 \%)$ were found to have ARDS at mechanical ventilation onset and 180 (10.9\%) developed ARDS after mechanical ventilation onset. The incidence of ARDS after mechanical ventilation onset was 1.07 events per $100 \mathrm{ICU}$ days (95\% CI 0.92 to 1.23 events per 100 ICU days). The average \pm SD number of days in the ICU before the onset of ARDS after mechanical ventilation onset was $6.5 \pm 6.2$ days. The majority of participants with ARDS after mechanical ventilation onset $(65 \%, n=$ 117) developed ARDS in $<7$ days of their ICU stay.

We summarize demographics, severity of disease, and past medical history by ARDS status in Table 1. Participants with ARDS at mechanical ventilation onset had a similar APACHE III $(p=0.63)$, SAPS II $(p=0.50)$, and SOFA $(p=0.37)$ at the initiation of mechanical ventilation than those with ARDS after mechanical ventilation onset. APACHE III $(p=0.75)$, SAPS II $(p=0.19)$, and SOFA $(p=0.98)$ at the initiation of mechanical ventilation were similar between those with ARDS after mechanical ventilation onset and no ARDS. On average, however, those with ARDS at any point during the study had a higher APACHE III $(p=0.05)$, SAPS II $(p=0.01)$, and SOFA $(p=0.01)$ scores than those who never developed ARDS. BMI was higher in participants with ARDS when compared to participants who never developed ARDS $(p<0.01)$, but similar between those who had ARDS at mechanical ventilation onset and those who developed ARDS after mechanical ventilation onset $(p=$ 0.18). Participants with ARDS after mechanical ventilation onset had a greater ABW-PBW than those who never developed ARDS $(p=0.01)$, whereas ABW-PBW was similar to those who had ARDS at mechanical ventilation onset $(p=0.16)$. Participants with ARDS at mechanical ventilation onset had a lower prevalence of congestive heart failure when compared to those with either in-ICU ARDS $(<0.01)$ or those who never developed ARDS $(p<0.01)$, whereas we did not find a difference between those who developed ARDS after mechanical ventilation onset and those who never developed ARDS $(p=0.51)$. Participants with ARDS at mechanical ventilation onset had a higher prevalence of having HIV when compared to those with either ARDS after mechanical ventilation onset or those who never developed ARDS $(p<0.01)$.

\section{Ventilator parameters at the initiation of mechanical ventilation}

We summarize ventilator parameters at the initiation of mechanical ventilation by ARDS status in Table 4. Average \pm SD tidal volume per kilogram of predicted body weight $(\mathrm{kg}$ PBW) for mechanical ventilation was $8.5 \pm$ $2.2 \mathrm{~mL} / \mathrm{kg}$ for the entire cohort. Among the participants with ARDS at mechanical ventilation onset, $89.6 \%(n=$ 295) were ventilated with tidal volumes $>6 \mathrm{~mL} / \mathrm{kg} \mathrm{PBW}$, whereas $92.6 \%(n=165)$ of those who developed ARDS after mechanical ventilation onset and 91.1\% $(n=1020)$ of those who never developed ARDS were initially ventilated with tidal volumes $>6 \mathrm{~mL} / \mathrm{kg}$ PBW. Plateau pressure was measured in $71 \%(n=1177)$ of individuals in this study. Although plateau pressure was $\leq 30 \mathrm{~cm} \mathrm{H}_{2} \mathrm{O}$ in a majority of participants $(83.5 \%)$ at the initiation of mechanical ventilation, $52.2 \%$ had driving pressures $>15$ $\mathrm{cm} \mathrm{H}_{2} \mathrm{O}$. Plateau pressure at the initiation of mechanical ventilation was higher in participants with ARDS on presentation when compared to others $(p<0.001)$. There was no difference in driving pressure at the initiation of mechanical ventilation between those with ARDS at mechanical ventilation onset and ARDS after mechanical ventilation onset $\left(17.4 \mathrm{~cm} \mathrm{H}_{2} \mathrm{O}\right.$ vs 16.4 $\mathrm{cm} \mathrm{H}_{2} \mathrm{O}, p=0.17$ ). While plateau and driving pressures at the initiation of mechanical ventilation were higher in participants with ARDS after mechanical ventilation onset than in those who never developed ARDS, this increase was not significant $(p=0.45$ and $p=0.45$, respectively). Individuals with ARDS on presentation had the highest average set PEEP at the initiation of mechanical ventilation when compared to those with inICU ARDS or those who never developed ARDS $(p<$ 
Table 1 Baseline characteristics comparing mechanically ventilated participants with ARDS at mechanical ventilation onset, those of developed ARDS after mechanical ventilation onset, and those who never developed ARDS

\begin{tabular}{|c|c|c|c|c|c|}
\hline Characteristic & Overall & $\begin{array}{l}\text { ARDS at mechanical } \\
\text { ventilation onset }\end{array}$ & $\begin{array}{l}\text { ARDS after mechanical } \\
\text { ventilation onset }\end{array}$ & No ARDS & $p$ value \\
\hline Number of participants, $n(\%)$ & $1657(100.0)$ & $334(20.2)$ & $180(10.9)$ & $1143(69.0)$ & \\
\hline Being male, $n(\%)$ & $907(54.7)$ & $191(57.2)$ & $92(51.1)$ & $624(54.6)$ & 0.41 \\
\hline Age in years, mean (SD) & $60.0(18.9)$ & $58.3(19.4)$ & $59.1(20.6)$ & $60.7(18.4)$ & 0.10 \\
\hline Body mass index in $\mathrm{kg} / \mathrm{m}^{2}$, mean (SD) & $26.7(5.3)$ & $27.0(5.4)$ & $27.7(5.6)$ & $26.5(5.2)$ & 0.02 \\
\hline Height in centimeters, mean (SD) & $161.6(8.8)$ & $161.3(8.3)$ & $161.5(9.5)$ & $161.8(8.8)$ & 0.68 \\
\hline $\begin{array}{l}\text { Difference between actual and predicted } \\
\text { body weight in } \mathrm{kg} \text {, mean (SD) }\end{array}$ & $13.7(14.0)$ & $14.2(14.3)$ & $16.1(15.0)$ & $13.2(13.8)$ & 0.04 \\
\hline \multicolumn{6}{|l|}{$\begin{array}{l}\text { Severity scores at the initiation of mechanical } \\
\text { ventilation, mean (SD) }\end{array}$} \\
\hline APACHE III & $82.7(28.1)$ & $85.9(28.6)$ & $82.5(28.9)$ & $81.8(27.7)$ & 0.07 \\
\hline SOFA & $9.5(3.5)$ & $10.1(3.3)$ & $9.3(3.6)$ & $9.3(3.5)$ & $<0.01$ \\
\hline SAPS $\|$ & $54.4(15.6)$ & $56.0(16.5)$ & $55.4(16.0)$ & $53.7(15.3)$ & 0.04 \\
\hline \multicolumn{6}{|l|}{ Comorbidities, $n$ (\%) } \\
\hline HIV & $29(1.8)$ & $14(4.2)$ & $0(0.0)$ & $15(1.3)$ & $<0.01$ \\
\hline Tuberculosis & $95(5.7)$ & $19(5.7)$ & $10(5.6)$ & $66(5.8)$ & 0.99 \\
\hline Leukemia & $15(0.9)$ & $6(1.8)$ & $2(1.1)$ & $7(0.6)$ & 0.12 \\
\hline Malignant tumor & $122(7.4)$ & $19(5.9)$ & $11(6.2)$ & $52(4.9)$ & 0.75 \\
\hline Dialysis & $122(7.4)$ & $16(4.8)$ & $11(6.1)$ & $95(8.3)$ & 0.07 \\
\hline COPD & $32(1.9)$ & $7(2.2)$ & $4(2.3)$ & $21(1.8)$ & 0.93 \\
\hline Cirrhosis & $63(3.8)$ & $17(5.2)$ & $8(4.5)$ & $38(3.3)$ & 0.15 \\
\hline Chronic renal disease & $251(15.2)$ & $43(13.3)$ & $21(11.8)$ & $187(16.2)$ & 0.43 \\
\hline Diabetes & $254(15.4)$ & $39(12.0)$ & $25(15.4)$ & $190(16.5)$ & 0.15 \\
\hline Congestive heart failure & $153(9.3)$ & $18(5.6)$ & $17(9.6)$ & $118(10.3)$ & 0.01 \\
\hline Vasopressor use first $24 \mathrm{~h}, n$ (\%) & $1002(60.5)$ & $1002(60.5)$ & $104(57.8)$ & $688(60.2)$ & 0.49 \\
\hline Fluid balance first $24 \mathrm{~h}, \mathrm{~mL}$ (SD) & $4474(2607)$ & $4160(2192)$ & $4170(2345)$ & $4515(2744)$ & $<0.01$ \\
\hline \multicolumn{6}{|l|}{ Admission service, $n(\%)$} \\
\hline Medicine & $1196(72.3 \%)$ & $240(73.8 \%)$ & $125(70.2 \%)$ & $831(72.1 \%)$ & 0.86 \\
\hline Trauma & $177(10.7 \%)$ & $25(7.7 \%)$ & $19(10.7 \%)$ & $133(11.5 \%)$ & 0.23 \\
\hline Scheduled surgery & $58(3.5 \%)$ & $14(4.3 \%)$ & $3(1.7 \%)$ & $41(3.6 \%)$ & 0.81 \\
\hline Unscheduled surgery & $178(10.8 \%)$ & $33(10.1 \%)$ & $28(15.7 \%)$ & $117(10.2 \%)$ & 0.75 \\
\hline Other & $46(2.8 \%)$ & $13(4 \%)$ & $3(1.7 \%)$ & $30(2.6 \%)$ & 0.58 \\
\hline
\end{tabular}

0.01). All airway pressures collected at the initiation of mechanical ventilation were higher in participants with ARDS at mechanical ventilation onset, followed by participants ARDS after mechanical ventilation onset and lowest in those who never developed ARDS (Table 2).

\section{Clinical outcomes}

Mortality at 90 days was $55.6 \%$ for study participants who had ARDS anytime during the study as compared to $46.0 \%$ in participants who never developed ARDS $(p<0.01)$. Participants with ARDS at mechanical ventilation onset had a similar 90-day mortality than that of participants with ARDS after mechanical ventilation onset in unadjusted (56\% vs $55 \% ; p=0.96)$ and adjusted analysis $(p=0.79)$. Although participants with ARDS at mechanical ventilation onset had a worse median survival than those with ARDS after mechanical ventilation onset ( 40 days vs 60 days, respectively), both groups had a similar 90-day survival (Fig. 1).

We plotted the cumulative percentage of mechanically ventilated participants who achieved unassisted breathing (including those who were discharged alive) and those who died after enrollment in the first 28 days, stratified by ARDS status (Fig. 2). Median time to unassisted breathing was 7 days in no ARDS, 10 days in ARDS at mechanical ventilation onset, and 12 days in ARDS after mechanical ventilation onset. Maximum convergence at 28 days of the cumulative incidences for 
death and achieving unassisted breathing was seen in those with no ARDS, whereas the least amount of convergence was seen those with ARDS after mechanical ventilation onset. This indicates that participants who developed ARDS after mechanical ventilation onset spent a higher proportion of their time on mechanical ventilation (Fig. 2).

Ventilator-free days, ICU-free days, and hospital-free days were lowest in those with ARDS after mechanical ventilation onset followed by those with ARDS at mechanical ventilation onset and highest in those who never developed ARDS both in single variable (Table 3) and adjusted analyses (Table 4).

\section{Factors associated with the development of ARDS after mechanical ventilation onset}

Individual risk factors associated with the risk of developing ARDS after mechanical ventilation onset included a higher BMI, a larger difference between actual and predicted body weight, a higher set PEEP, higher mean airway pressure, higher peak inspiratory airway pressures, and a lower fluid balance in the first $24 \mathrm{~h}$ (Fig. 3). In adjusted analyses, fluid balance in the first $24 \mathrm{~h}$ was no longer significant (interquartile $\mathrm{OR}=0.92,95 \% \mathrm{CI}$ 0.74-1.14), whereas BMI (interquartile OR $=1.18,95 \%$ CI 1.01-1.38) and the difference between actual and predicted body weight (interquartile $\mathrm{OR}=1.20,95 \% \mathrm{CI}$ 1.03-1.39) remained independent predictors for the development of ARDS after mechanical ventilation onset. However, we did not find a difference in tidal volumes at the initiation of mechanical ventilation between participants who developed ARDS following initiation of mechanical and those never developed ARDS. Based on these findings, we tested if tidal volumes at the initiation of mechanical ventilation varied with BMI and predicted body weight. In these analyses, however, we found that tidal volumes at the initiation of mechanical ventilation were not associated with BMI but were positively associated with predicted body weight (Additional file 1: Fig. S3).

In adjusted analyses, mean airway pressure, peak airway pressures, and PEEP at the initiation of mechanical ventilation remained significantly associated with the development of ARDS after mechanical ventilation onset (Fig. 4). We also compared risk factors between developed early and late ARDS following initiation of mechanical ventilation, but did not find any differences in age, sex, baseline severity of illness scores, baseline ventilator parameters, and outcomes including 90-day mortality, ventilator-free days, hospital-free days, and ICU-free days in between the two groups in single variable analysis or (Additional file 1: Table S1) adjusted analyses (Additional file 1: Table S2).

\section{Discussion}

In this multicenter study of mechanically ventilated participants in Peru, nearly a third of study participants either had ARDS at mechanical ventilation onset (20.2\%) or developed ARDS after the onset of mechanical ventilation (10.9\%). A large observational study (LUNG SAFE) conducted in 459 ICUs in 50 countries reported a similar incidence of ARDS during a 4-week period: $23.4 \%$ of all patients requiring mechanical ventilation with an incidence density of 1.5 cases of ARDS per 100 ICU days [29]. In another study conducted in a UK university hospital, the incidence of ARDS in patients admitted to the ICU over a 6 -month period was $13 \%$, and hospital mortality in patients with ARDS was $42 \%$ when compared to $11 \%$ in those without ARDS [30]. However, the UK study examined all patients admitted to an ICU whereas LUNG SAFE and our study examined the subset of patients who received mechanical ventilation.

Table 2 Ventilatory parameters at the initiation of mechanical ventilation among mechanically ventilated participants who had ARDS at mechanical ventilation onset, those who developed ARDS after mechanical ventilation onset, and those who never developed ARDS

\begin{tabular}{|c|c|c|c|c|c|}
\hline Variables, mean (SD) & $\begin{array}{l}\text { Overall, } \\
n=1657\end{array}$ & $\begin{array}{l}\text { ARDS at mechanical } \\
\text { ventilation onset, } n=334\end{array}$ & $\begin{array}{l}\text { ARDS after mechanical } \\
\text { ventilation onset, } n=180\end{array}$ & $\begin{array}{l}\text { No ARDS, } \\
n=1143\end{array}$ & $p$ value \\
\hline $\mathrm{FiO}_{2}$ & $0.47(0.18)$ & $0.54(0.19)$ & $0.44(0.15)$ & $0.45(0.18)$ & $<0.001$ \\
\hline PEEP, $\mathrm{cm} \mathrm{H}_{2} \mathrm{O}$ & $7.7(3.7)$ & $10.1(4.5)$ & $7.5(3.4)$ & $7.0(3.2)$ & $<0.001$ \\
\hline $\mathrm{PaO}_{2} / \mathrm{FiO}_{2}, \mathrm{mmHg}$ & $260.3(125.7)$ & $201.6(101.5)$ & $262.4(135.0)$ & $278.2(125.5)$ & $<0.001$ \\
\hline Tidal volume, $\mathrm{mL}$ & $470.9(98.9)$ & $479.2(108.7)$ & $468.4(93.0)$ & $468.9(96.7)$ & 0.23 \\
\hline Tidal volume, $\mathrm{mL} / \mathrm{kg}$ PBW & $8.5(2.2)$ & $8.7(2.3)$ & $8.6(2.1)$ & $8.5(2.2)$ & 0.16 \\
\hline Static compliance, $\mathrm{mL} / \mathrm{cm} \mathrm{H}_{2} \mathrm{O}$ & $34.8(23.9)$ & $33.9(20.4)$ & $34.7(22.1)$ & $35.1(25.2)$ & 0.79 \\
\hline Driving pressure, $\mathrm{cm} \mathrm{H}_{2} \mathrm{O}$ & $16.4(5.4)$ & $17.4(5.8)$ & $16.4(5.3)$ & $16.1(5.2)$ & $<0.01$ \\
\hline Mean airway pressure, $\mathrm{cm} \mathrm{H}_{2} \mathrm{O}$ & $12.6(4.2)$ & $15.2(4.6)$ & $12.6(4.0)$ & $11.9(3.8)$ & $<0.001$ \\
\hline Peak inspiratory pressure, $\mathrm{cm} \mathrm{H}_{2} \mathrm{O}$ & $25.1(6.7)$ & $29.2(7.0)$ & $25.4(6.7)$ & $23.9(6.1)$ & $<0.001$ \\
\hline Plateau pressure, $\mathrm{cm} \mathrm{H}_{2} \mathrm{O}$ & $24.0(6.7)$ & $27.2(7.5)$ & $23.5(6.0)$ & $23.1(6.3)$ & $<0.001$ \\
\hline
\end{tabular}




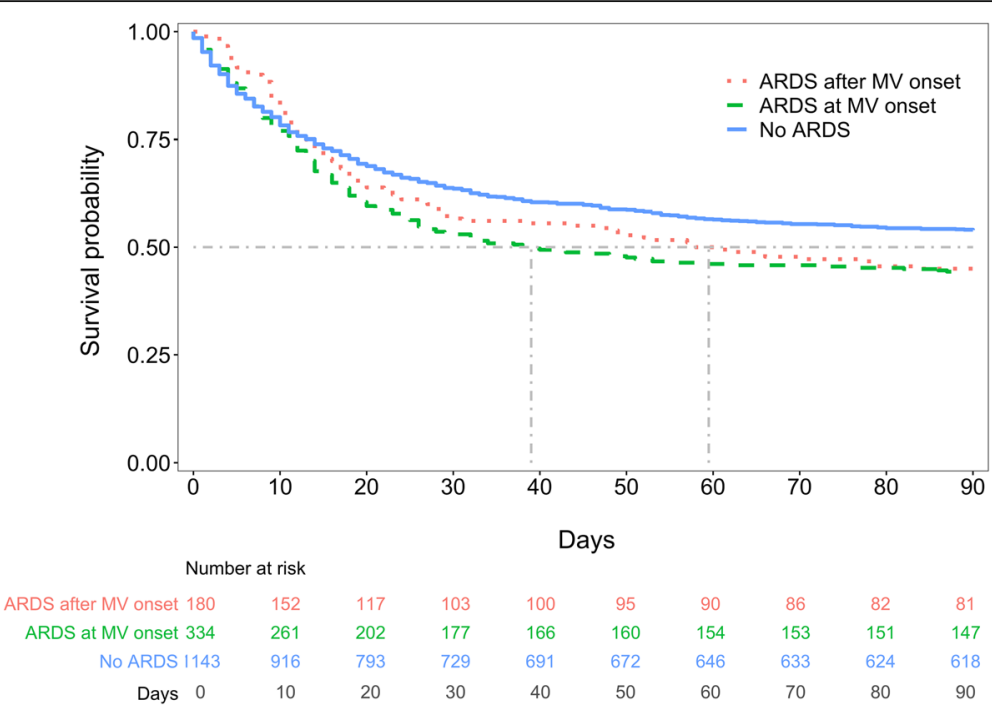

Fig. 1 Probability of survival by time in the ICU stratified by ARDS status. Those with no ARDS, shown in blue, had better 90-day survival than those with ARDS at mechanical ventilation [MV] onset (green) or ARDS after mechanical ventilation [MV] onset (red). The participants with ARDS on presentation had a worse survival median survival as compared to those with in-ICU ARDS (40 days vs 60 days) but similar 90-day survival

The overall 90-day mortality in our cohort of mechanically ventilated patients was high (49\%). Similar mortality rates have been reported in other studies in LMICs [31-36]. 90-day mortality was higher in those with ARDS at mechanical ventilation onset and those with ARDS after mechanical ventilation onset when compared to those who never developed ARDS. We also observed that the majority of study participants were ventilated with $>6 \mathrm{~mL} / \mathrm{kg}$ PBW. Indeed, the mean tidal volume for patients with ARDS at mechanical ventilation onset was $8.7 \mathrm{~mL} / \mathrm{kg}$ PBW. This is not in compliance with the ARDS Network trial recommendations of lungprotective ventilation with a tidal volume of $\leq 6 \mathrm{~mL} / \mathrm{kg}$ predicted body weight and a plateau pressure of $30 \mathrm{~cm}$ of $\mathrm{H}_{2} \mathrm{O}$ [37] and highlights an area for improvement. In routine clinical practice, however, non-compliance with ARDS Network ventilation protocols has also been commonly reported in studies conducted in the USA. For example, an observational study conducted in 57 ICUs in the USA found a compliance of $50 \%$ with tidal volumes $\leq 6.5 \mathrm{~mL} / \mathrm{kg}$ PBW in patients with ARDS [38]. In another observational study conducted in Maryland, USA, only $41 \%$ of ventilator settings in patients with ARDS were adherent to ARDS network goals [39]. Obstacles to the implementation of lung-protective ventilation need further elaboration in both resource-rich and resourcelimited settings to optimize clinical outcomes.

Another area for improvement is lung recruitment. While average peak and plateau pressures were $<30$ $\mathrm{cm} \mathrm{H}_{2} \mathrm{O}$, average driving pressures were $>15 \mathrm{~cm} \mathrm{H}_{2} \mathrm{O}$.
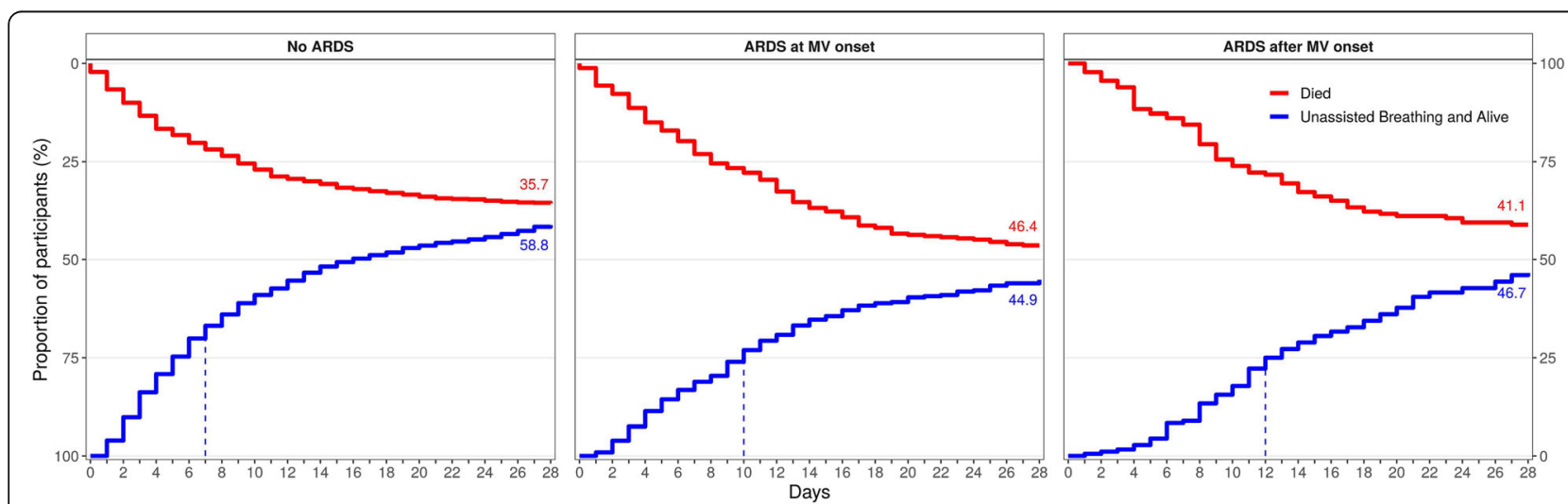

Fig. 2 Cumulative incidences of achieving unassisted breathing including being discharged alive and death in the 28-day period of follow-up among the three categories of participants: with no ARDS, ARDS at mechanical ventilation [MV] onset, and who developed ARDS after mechanical ventilation [MV] onset from left to right 
Table 3 Outcomes among study participants who had ARDS at mechanical ventilation onset, those who developed ARDS after mechanical ventilation onset, and those with no ARDS during their hospital stay

\begin{tabular}{|c|c|c|c|c|c|}
\hline & $\begin{array}{l}\text { Overall, } \\
n=1657\end{array}$ & $\begin{array}{l}\text { ARDS at mechanical } \\
\text { ventilation onset, } n=334\end{array}$ & $\begin{array}{l}\text { ARDS after mechanical } \\
\text { ventilation onset, } n=180\end{array}$ & $\begin{array}{l}\text { No ARDS, } \\
n=1143\end{array}$ & $p$ value \\
\hline Death 90 days, $n$ (\%) & $810(48.9 \%)$ & $187(56.0 \%)$ & $99(55.3 \%)$ & $524(46.0 \%)$ & $<0.01$ \\
\hline Ventilator-free days, mean (SD) & $10.1(10.7)$ & $7.7(10.0)$ & $6.4(8.6)$ & $11.3(10.9)$ & $<0.001$ \\
\hline ICU-free days, mean (SD) & $7.3(8.9)$ & $5.5(8.1)$ & $4.0(6.6)$ & $8.4(9.2)$ & $<0.001$ \\
\hline Hospital-free days, mean (SD) & $11.1(16.6)$ & $9.8(16.1)$ & $7.3(13.8)$ & $12.1(17.1)$ & $<0.001$ \\
\hline
\end{tabular}

This can be due to less than optimal lung recruitment, i.e., inadequate application of PEEP. The average set PEEP in this cohort of mechanically ventilated patients was $7.7 \mathrm{~cm} \mathrm{H}_{2} \mathrm{O}$. Although PEEP was higher in those with ARDS on presentation, higher levels of PEEP may actually be needed given high driving pressures. Lower driving pressure, irrespective of tidal volume, is associated with lower mortality in ARDS [40]. Therefore, individualized titration of PEEP through measurement of driving pressure and plateau pressure may improve clinical outcomes [41]. This along with low tidal volume ventilation can facilitate achieving lower driving pressures. While driving pressure at the initiation of mechanical ventilation was not associated with the development of ARDS after mechanical ventilation onset in our study, it is a known predictor of mortality in ARDS [29, 42].

We identified potential factors at the initiation of mechanical ventilation which were associated with the development of ARDS after mechanical ventilation onset. Among the ventilator-related risk factors, higher mean airway pressures, higher peak airway pressures, and higher set PEEP at the initiation of mechanical ventilation were all associated with a higher incidence of ARDS after mechanical ventilation onset in adjusted analyses. The association between higher airway pressures (peak, mean airway, and PEEP) and ARDS development could reflect lung injury, but it could also reflect lesscompliant lungs that are sicker and more prone to ARDS development. Indeed, high airway pressures have been linked with early barotrauma and higher mortality [43]. In fact, peak inspiratory airway pressure was also identified to be a potential target to lower mortality in an ancillary analysis from the LUNG SAFE study [43]. High inspiratory flow, for a given plateau pressure or strain, may contribute to ventilator-induced lung injury likely by locally intensified concentration of stress $[44,45]$. Higher mean airway pressures may also reflect patients with higher airway resistance, lower compliance, and more elevated respiratory rates which could reflect a greater dead space, all of which can identify a group of sicker patients who are more likely to get ARDS. Mean airway pressure reflects mean alveolar pressure throughout an entire respiratory cycle, which may better reflect the power applied to the lung and risk for injury throughout tidal breathing. Targeting peak or mean airway pressures, therefore, may play a role in preventing ARDS after mechanical ventilation onset.

In contrast, we did not find an association between the development of ARDS after mechanical ventilation onset and either plateau pressures or driving pressures at the initiation of mechanical ventilation, two well-described predictors of ARDS mortality. Nonetheless, both plateau pressure and driving pressure at the initiation of mechanical ventilation were higher in patients with ARDS at the start of mechanical when compared to those who developed ARDS after mechanical ventilation onset or never developed ARDS. There are a couple of possibilities that may explain why we did not find an association between the development of ARDS after mechanical ventilation onset and either plateau of driving pressures at the initiation of mechanical ventilation. First, plateau

Table 4 Regression results from analyses looking at differences in outcomes (death at 90 days, ventilator days, ICU-free days, and hospital-free days) by ARDS status

\begin{tabular}{llll}
\hline & $\begin{array}{l}\text { ARDS at mechanical ventilation } \\
\text { onset vs never developed ARDS }\end{array}$ & $\begin{array}{l}\text { ARDS after mechanical ventilation } \\
\text { onset vs never developed ARDS }\end{array}$ & $\begin{array}{l}\text { ARDS after mechanical ventilation onset vs } \\
\text { ARDS at mechanical ventilation onset }\end{array}$ \\
\hline $\begin{array}{l}\text { Odds ratio of death at } 90 \\
\text { days }(95 \% \mathrm{Cl})\end{array}$ & $1.61(1.21,2.14)$ & $1.54(1.07,2.20)$ & $0.95(0.63,1.45)$ \\
$\begin{array}{l}\text { Mean difference in } \\
\text { ventilator-free days }(95 \% \mathrm{Cl})\end{array}$ & $-3.82(-5.19,-2.44)$ & $-5.57(-7.32,-3.82)$ & $-1.75(-3.78,0.28)$ \\
$\begin{array}{l}\text { Mean difference in ICU- } \\
\text { free days }(95 \% \mathrm{Cl})\end{array}$ & $-2.95(-4.09,-1.81)$ & $-5.99(-6.43,-3.54)$ & $-2.04(-3.72,-036)$ \\
$\begin{array}{l}\text { Mean difference in } \\
\text { hospital-free days }(95 \% \mathrm{Cl})\end{array}$ & $-3.04(-5.21,-0.87)$ & $-3.05(-6.03,-0.08)$ \\
\hline
\end{tabular}




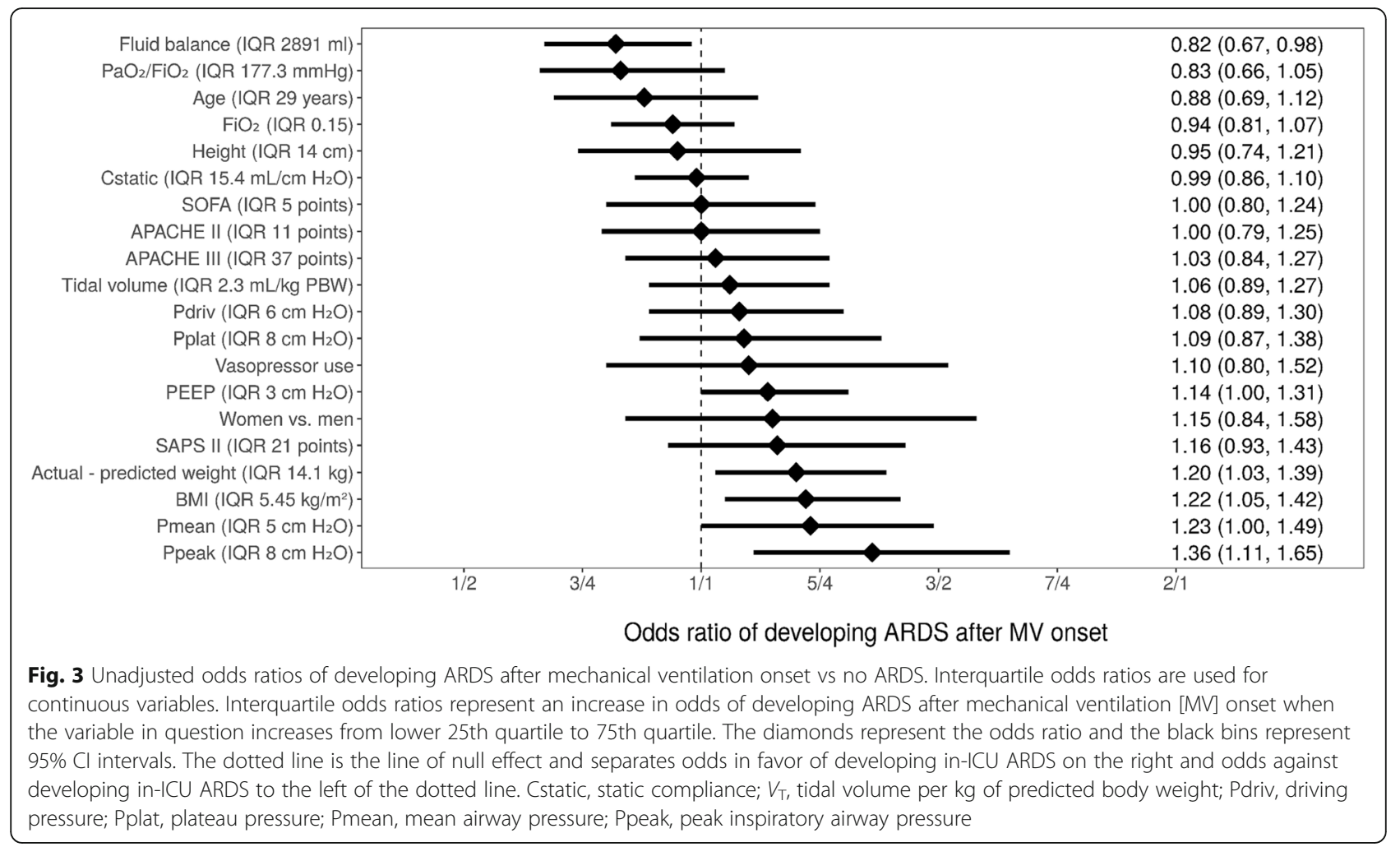

or driving pressure may take longer to rise in relation to the time of onset of ARDS compared to peak or mean airway pressure. Therefore, plateau or driving pressures measured at the initiation of mechanical ventilation may not capture this relationship. Mean or peak airway pressures may be elevated earlier than plateau or driving pressures in patients who go on to develop ARDS later in the course of mechanical ventilation because physicians may set a higher inspiratory flow rate in response to compensatory tachypnea commonly observed in mechanically ventilated patients with metabolic acidosis resulting from sepsis or septic shock, pancreatitis, or

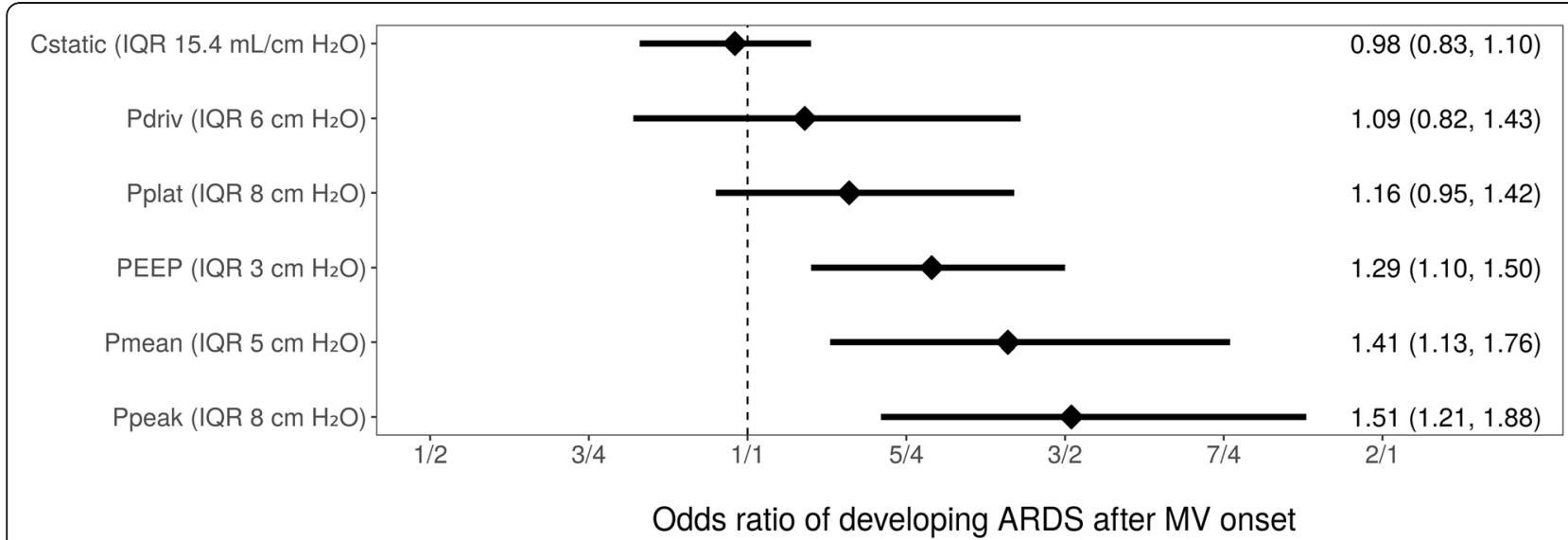

Fig. 4 Adjusted odds ratios of developing ARDS after mechanical ventilation onset as compared to no ARDS. All the variables shown were analyzed in separate multivariable models to avoid collinearity. All regression models were adjusted for age, sex, hospital, PEEP, and BMI. Interquartile odds ratios are used for continuous variables. Interquartile odds ratios represent an increase in odds of developing ARDS after mechanical ventilation [MV] onset when the variable in question increases from the lower 25 th to the 75 th percentile. The diamonds represent the odds ratio, and the black bins represent $95 \% \mathrm{Cl}$ intervals. The dotted line is the line of null effect and separates odds in favor of developing ARDS after mechanical ventilation [MV] onset to the right and odds against developing ARDS after mechanical ventilation [MV] onset to the left of the dotted line. Cstatic, static compliance; Pdriv, driving pressure; Pplat, plateau pressure; Pmean, mean pressure; Ppeak, peak pressure 
trauma, all of which are well-known risk factors for ARDS. A set higher inspiratory flow rate will result in higher peak airway pressures whereas plateau or driving pressures are minimally affected [46]. Patients with sepsis may also have circulatory inflammatory mediators which causes bronchoconstriction and consequently higher airway resistance [47-49]. This also results in higher peak airway pressures whereas plateau or driving pressures are minimally affected. Patients may also have pulmonary edema that precedes radiographic changes before it causes flooding of alveoli, but which affects conducting airways and increases airway resistance [50]. Second, we had fewer measurements of plateau or driving pressures when compared to peak or mean airway pressure which could have affected our ability to measure a statistically significant difference. We may have had fewer measurements of plateau pressure or driving pressure because they can be cumbersome or difficult to achieve, as it requires a half second inspiratory hold on a ventilator to calculate driving pressure. In our study, nearly $30 \%$ (480) of the patients were missing a plateau pressure recording at the initiation of mechanical ventilation. Similarly, in the multinational LUNG SAFE study, plateau pressure was available in only $40 \%$ of patients with ARDS, again showing a deficiency of measurement of plateau pressure worldwide in the management of ARDS [29]. It is well established from clinical and animal studies that presumptive application of protective ventilation with low tidal volume to keep plateau pressure $<30 \mathrm{~cm} \mathrm{H}_{2} \mathrm{O}$ can reduce the incidence of ARDS [51, 52].

Among patient-related risk factors, higher BMI in participants who did not have ARDS on presentation was significantly associated with the development of ARDS after mechanical ventilation onset. The association between BMI and development of ARDS after mechanical ventilation onset was not to differences in tidal volume. However, it was associated with a statistically significant difference between actual and predicted body weight. This suggests that excess adipose tissue in obese individuals may predispose them to an elevated risk for developing ARDS. Several mechanisms have been proposed for this association in previously published studies like obesity-induced adipokine imbalance [53], obesityinduced endoplasmic reticulum stress-causing endothelium dysfunction [54], chronic inflammatory state of obesity, and elevated neutrophils and cytokines that induce upregulation of adhesion molecules on lung epithelium and enhanced susceptibility to injury [55]. This association has previously been demonstrated in both animal and human studies and is not explained by initial ventilator settings in these individuals.

Our study has some potential shortcomings. First, both higher airway pressure at mechanical ventilation onset and subsequent development of ARDS after mechanical ventilation onset could reflect an underlying severe lung injury rather than a causal effect. Regardless, they serve as an indicator of the development of ARDS in the future, in a cohort of patients who initially do not meet the criterion for ARDS and hence provide an opportunity for early intervention. In addition, the association was adjusted for baseline severity of illness score APACHE III accounting for some of the disease severity at presentation. Second, our study was designed before the publication of the Berlin definition, but the collection of necessary information allowed for the addition of parameters like PEEP to the existing definition to make the criteria more aligned with the Berlin definition. Third, we did not have information about the potential etiology of injury that could have led to the development of ARDS. This precluded us from calculating a Lung Injury Prevention Score (LIPS) at baseline which has been studied to identify those at risk for acute lung injury [56]. Choosing mechanically ventilated participants helped us to enrich our cohort with individuals who are at the highest risk of developing ARDS and enabled us to isolate iatrogenic risk factors in mechanically ventilated individuals. Overall, the observational design allowed us to study current practices and incidence of disease and identify gaps and areas of improvement. Fourth, because this is an observational study, we did not dictate how frequently physicians obtained chest X-rays or arterial blood gases while on mechanical ventilation. This may result in an underestimation of the ARDS incidence after mechanical ventilation onset. Finally, our study was conducted between 2010 and 2013, and changes in the management of sepsis and mechanical ventilation could affect how our findings are applicable today. Replication of our findings in more recent cohorts is necessary.

\section{Conclusions}

ARDS after the onset of mechanical ventilation is common in mechanically ventilated adults, and the associated mortality is equally high as those who are initially admitted with ARDS. Hospital-free days and ICU-free days were lowest among patients with ARDS after mechanical ventilation onset. In this cohort of mechanically ventilated patients in Peru, optimal lung protection ventilation was not used and driving pressures were commonly high. Risk factors for the development of ARDS after mechanical ventilation onset include a high body mass index and the presence of high airway pressures (peak, mean airway, and positive end-expiratory pressures) early in the course of mechanical ventilation. Future studies need to validate the use of these early factors to identify mechanically ventilated patients at risk for developing ARDS after initiation of mechanical ventilation. 


\section{Supplementary information}

Supplementary information accompanies this paper at https://doi.org/10. 1186/s13054-019-2646-8.

Additional file 1: Table S1. Baseline Characteristics, ventilatory parameters and outcomes in participants with early ARDS ( $<7$ days) and late ARDS ( $\geq 7$ days) after mechanical ventilation onset. Table S2. Adjusted outcomes among study participants comparing early ARDS $(<7$ days) and late ARDS ( $\geq 7$ days) after mechanical ventilation onset. All regression models are adjusted for age, sex, hospital and APACHE III. Figure S1. Study Flowchart. Figure S2. Percentage of ICU-days when a chest radiograph and an arterial blood gas were obtained for all days after mechanical ventilation onset. Figure S3. Scatter plot of tidal volume (y-axis) and body mass index (x-axis) on the left and tidal volume (y-axis) and predicted body weight ( $\mathrm{x}$-axis) on the right.

\section{Abbreviations}

ARDS: Acute respiratory distress syndrome; ICU: Intensive care unit; LMICs: Low- and middle-income countries; BMI: Body mass index; PEEP: Positive end-expiratory pressure; AUC: Area under the receiver operating characteristic curves; APACHE: Acute Physiology and Chronic Health Evaluation; SOFA: Sequential Organ Failure Assessment; SAPS: Simplified Acute Physiology Score; HIV: Human immunodeficiency virus; PBW: Predicted body weight; LIPS: Lung Injury Prevention Score

\section{Acknowledgements}

We thank the nurses and staff at all four participating hospitals and at A.B. PRISMA who assisted us in the completion of this study. We are also grateful to Dr. Joshua Denney, Dr. Long Davalos, Dr. Francesca Capanni, and Dr. Augusto Dulanto for their help in the supervision of this study. INTENSIVOS Cohort Study Group: Roy G Brower, Francesca Capanni, Maria A Caravedo, Jorge Cerna, William Checkley, Eduardo E Chirinos, Long Davalos, Aldo De Ferrari, Joshua A Denney, Augusto Dulanto, Phabiola Herrera, Amador A Jaymez, Nicole Mongilardi, Carmen Paredes, Enrique Paz, Maria Alejandra Pereda, Jose Portugal, Rocio Quispe, Rollin Roldan, Navid Shams.

\section{Authors' contributions}

EG and WC were equally responsible for the writing of the manuscript. SH and MRG conducted the statistical analyses. WC, PH, RR, EP, AAJ, EEC, JP, and $\mathrm{RQ}$ participated in the study design and conduct and assisted in the writing of the manuscript. RGB provided expert guidance in the design and conduct of this study and assisted in the writing of the manuscript. Each author made substantial contributions to the conception or design of the work, the acquisition, analysis or interpretation of data, and drafting and final approval of the manuscript. All authors read and approved the final manuscript. WC conceived the study and had ultimate oversight for the design and conduct and writing of this manuscript.

\section{Funding}

This study was supported by a Pathway to Independence Award (R00HL096955) from the National Heart, Lung and Blood Institute, United States National Institutes of Health.

\section{Availability of data and materials}

The datasets used and/or analyzed during the current study are available from the corresponding author on request.

\section{Ethics approval and consent to participate}

We received ethics approval and permission to conduct this study in each of the participating institutions: Hospital Nacional Edgardo Rebagliati Martins, Hospital Nacional Guillermo Almenara Irigoyen, Hospital Nacional Arzobispo Loayza, and Hospital de Emergencias Casimiro Ulloa. Ethics approvals were obtained from the institutional review boards of A.B. PRISMA and ESSALUD Hospital Nacional Edgardo Rebagliati Martins in Lima, Peru, and the Johns Hopkins School of Medicine in Baltimore, USA. We obtained a waiver of written informed consent from all institutions to conduct this observational study.

\section{Consent for publication}

We obtained consent for publication of de-identified data.

\section{Competing interests}

The authors declare that they have no competing interests.

\section{Author details}

${ }^{1}$ Division of Pulmonary and Critical Care, School of Medicine, Johns Hopkins University, 1830 E. Monument St, Room 555, Baltimore, MD 21287, USA.

${ }^{2}$ Center for Global Non-Communicable Disease Research and Training, School of Medicine, Johns Hopkins University, Baltimore, USA. ${ }^{3}$ Servicio de Cuidados Intensivos, Hospital Nacional Edgardo Rebagliati Martins, Lima, Peru. ${ }^{4}$ Servicio de Cuidados Intensivos, Hospital Nacional Guillermo Almenara Irigoyen, Lima, Peru. ${ }^{5}$ Servicio de Cuidados Intensivos, Hospital Nacional Arzobispo Loayza, Lima, Peru. ${ }^{6}$ Servicio de Cuidados Intensivos, Hospital De Emergencias José Casimiro Ulloa, Lima, Peru.

Received: 6 March 2019 Accepted: 9 October 2019 Published online: 06 December 2019

\section{References}

1. Task Force ARDS, Ranieri VM, Rubenfeld GD, Thompson BT, Ferguson ND, Caldwell E, et al. Acute respiratory distress syndrome: the Berlin definition. JAMA. 2012;307(23):2526-33.

2. MacCallum NS, Evans TW. Epidemiology of acute lung injury. Curr Opin Crit Care. 2005;11(1):43-9.

3. Rubenfeld GD, Caldwell E, Peabody E, Weaver J, Martin DP, Neff M, et al. Incidence and outcomes of acute lung injury. N Engl J Med. 2005;353(16):1685-93.

4. Bersten AD, Edibam C, Hunt T, Moran J, Australian, Group NZICSCT. Incidence and mortality of acute lung injury and the acute respiratory distress syndrome in three Australian states. Am J Respir Crit Care Med. 2002;165(4):443-8

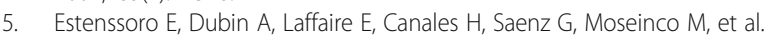
Incidence, clinical course, and outcome in 217 patients with acute respiratory distress syndrome. Crit Care Med. 2002;30(11):2450-6.

6. Villar J, Blanco J, Anon JM, Santos-Bouza A, Blanch L, Ambros A, et al. The ALIEN study: incidence and outcome of acute respiratory distress syndrome in the era of lung protective ventilation. Intensive Care Med. 2011;37(12): 1932-41.

7. Laffey JG, Madotto F, Bellani G, Pham T, Fan E, Brochard L, et al. Geoeconomic variations in epidemiology, patterns of care, and outcomes in patients with acute respiratory distress syndrome: insights from the LUNG SAFE prospective cohort study. Lancet Respir Med. 2017;5(8):627-38.

8. Brochard L, Roudot-Thoraval F, Roupie E, Delclaux C, Chastre J, Fernandez-

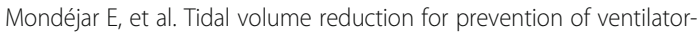
induced lung injury in acute respiratory distress syndrome. The multicenter trial group on tidal volume reduction in ARDS. Am J Respir Crit Care Med. 1998;158(6):1831-8.

9. Brower RG, Lanken PN, MacIntyre N, Matthay MA, Morris A, Ancukiewicz M, Schoenfeld D, Thompson BT; National Heart, Lung, and Blood Institute ARDS Clinical Trials Network. Higher versus lower positive end-expiratory pressures in patients with the acute respiratory distress syndrome. N Eng J Med. 2004:351(4):327-36.

10. Mercat A, Richard JM, Vielle B, et al. Positive end-expiratory pressure setting in adults with acute lung injury and acute respiratory distress syndrome: a randomized controlled trial. JAMA. 2008;299(6):646-55.

11. Levy MM. PEEP in ARDS--how much is enough? N Engl J Med. 2004;351(4): 389-91.

12. National Heart, Lung, and Blood Institute Acute Respiratory Distress Syndrome (ARDS) Clinical Trials Network, Wiedemann HP, Wheeler AP, Bernard GR, Thompson BT, Hayden D, deBoisblanc B, Connors AF Jr, Hite $\mathrm{RD}$, Harabin AL. Comparison of two fluid-management strategies in acute lung injury. N Eng J Med. 2006;354(24):2564-75.

13. Guérin C, Reignier J, Richard J-C, Beuret P, Gacouin A, Boulain T, et al. Prone positioning in severe acute respiratory distress syndrome. N Engl J Med. 2013;368(23):2159-68.

14. National Heart, Lung, and Blood Institute ARDS Clinical Trials Network Truwit JD, Bernard GR, Steingrub J, Matthay MA, Liu KD, Albertson TE, Brower RG, Shanholtz C, Rock P, Douglas IS, deBoisblanc BP, Hough CL, Hite $\mathrm{RD}$, Thompson BT. Rosuvastatin for sepsis-associated acute respiratory distress syndrome. N Eng J Med. 2014;370(23):2191-200.

15. Meduri GU, Golden E, Freire AX, Taylor E, Zaman M, Carson SJ, et al. Methylprednisolone infusion in early severe ARDS: results of a randomized controlled trial. Chest. 2007;131(4):954-63. 
16. Riviello ED, Pisani $L$, Schultz MJ. What's new in ARDS: ARDS also exists in resource-constrained settings. Intensive Care Med. 2016;42(5):794-6.

17. Crisp N, Chen L. Global supply of health professionals. N Engl J Med. 2014; 370(10):950-7.

18. Tripathi S, Kaur H, Kashyap R, Dong Y, Gajic O, Murthy S. A survey on the resources and practices in pediatric critical care of resource-rich and resource-limited countries. J Intensive Care. 2015;3:40.

19. Murthy S, Leligdowicz A, Adhikari NK. Intensive care unit capacity in lowincome countries: a systematic review. PLoS One. 2015;10(1):e0116949.

20. Mehta AB, Syeda SN, Wiener RS, Walkey AJ. Epidemiological trends in invasive mechanical ventilation in the United States: a population-based study. J Crit Care. 2015;30(6):1217-21.

21. Li G, Malinchoc M, Cartin-Ceba R, Venkata CV, Kor DJ, Peters SG, et al. Eight-year trend of acute respiratory distress syndrome: a populationbased study in Olmsted County, Minnesota. Am J Respir Crit Care Med. 2011;183(1):59-66.

22. Denney JA, Capanni F, Herrera P, Dulanto A, Roldan R, Paz E, et al. Establishment of a prospective cohort of mechanically ventilated patients in five intensive care units in Lima, Peru: protocol and organisational characteristics of participating centres. BMJ Open. 2015;5(1):e005803.

23. Jones AE, Trzeciak S, Kline JA. The Sequential Organ Failure Assessment score for predicting outcome in patients with severe sepsis and evidence of hypoperfusion at the time of emergency department presentation. Crit Care Med. 2009;37(5):1649-54.

24. Le Gall JR, Lemeshow S, Saulnier F. A new Simplified Acute Physiology Score (SAPS II) based on a European/North American multicenter study. JAMA. 1993;270(24):2957-63.

25. Knaus WA, Wagner DP, Draper EA, Zimmerman JE, Bergner M, Bastos PG, et al. The APACHE III prognostic system. Risk prediction of hospital mortality for critically ill hospitalized adults. Chest. 1991;100(6):1619-36.

26. Zhi G, Xin W, Ying W, Guohong X, Shuying L. "Obesity paradox" in acute respiratory distress syndrome: a systematic review and meta-analysis. PLoS ONE. 2016;11(9):e0163677.

27. Determann RM, Royakkers A, Wolthuis EK, Vlaar AP, Choi G, Paulus F, et al Ventilation with lower tidal volumes as compared with conventional tidal volumes for patients without acute lung injury: a preventive randomized controlled trial. Crit Care. 2010;14(1):R1.

28. Serpa Neto A, Cardoso SO, Manetta JA, Pereira VG, Esposito DC, Pasqualucci Mde $\mathrm{O}$, et al. Association between use of lung-protective ventilation with lower tidal volumes and clinical outcomes among patients without acute respiratory distress syndrome: a meta-analysis. JAMA. 2012;308(16):1651-9.

29. Bellani G, Laffey JG, Pham T, et al. Epidemiology, patterns of care, and mortality for patients with acute respiratory distress syndrome in intensive care units in 50 countries. JAMA. 2016;315(8):788-800.

30. Summers C, Singh NR, Worpole L, Simmonds R, Babar J, Condliffe AM, et al. Incidence and recognition of acute respiratory distress syndrome in a UK intensive care unit. Thorax. 2016:71(11):1050-1.

31. Karthikeyan B, Kadhiravan T, Deepanjali S, Swaminathan RP. Case-mix, care processes, and outcomes in medically-ill patients receiving mechanical ventilation in a low-resource setting from southern India: a prospective clinical case series. PLoS One. 2015;10(8):e0135336.

32. Pisani L, Algera AG, Serpa Neto A, Ahsan A, Beane A, Chittawatanarat K, et al. PRactice of VENTilation in Middle-Income Countries (PRoVENT-iMIC): rationale and protocol for a prospective international multicentre observational study in intensive care units in Asia. BMJ Open. 2018;8(4):e020841.

33. Azevedo LC, Park M, Salluh Jl, Rea-Neto A, Souza-Dantas VC, Varaschin P, et al. Clinical outcomes of patients requiring ventilatory support in Brazilian intensive care units: a multicenter, prospective, cohort study. Crit Care. 2013; 17(2):R63.

34. Epidemiology, practice of ventilation and outcome for patients at increased risk of postoperative pulmonary complications: LAS VEGAS - an observational study in 29 countries. Eur J Anaesthesiol. 2017;34(8):492-507.

35. Esteban A, Frutos-Vivar F, Muriel A, Ferguson ND, Penuelas O, Abraira V, et al. Evolution of mortality over time in patients receiving mechanical ventilation. Am J Respir Crit Care Med. 2013;188(2):220-30.

36. Neto AS, Barbas CSV, Simonis FD, Artigas-Raventos A, Canet J, Determann RM, et al.; PRoVENT; PROVE Network investigators. Epidemiological characteristics, practice of ventilation, and clinical outcome in patients at risk of acute respiratory distress syndrome in intensive care units from 16 countries (PRoVENT): an international, multicentre, prospective study. Lancet Respir Med. 2016;4(11):882-93.
37. Brower RG, Matthay MA, Morris A, Schoenfeld D, Thompson BT, Wheeler A. Ventilation with lower tidal volumes as compared with traditional tidal volumes for acute lung injury and the acute respiratory distress syndrome. N Engl J Med. 2000;342(18):1301-8.

38. Sevransky JE, Checkley W, Herrera P, Pickering BW, Barr J, Brown SM, et al. Protocols and hospital mortality in critically ill patients: the United States critical illness and injury trials group critical illness outcomes study. Crit Care Med. 2015;43(10):2076-84.

39. Needham DM, Colantuoni E, Mendez-Tellez PA, Dinglas VD, Sevransky JE, Dennison Himmelfarb CR, et al. Lung protective mechanical ventilation and two year survival in patients with acute lung injury: prospective cohort study. BMJ. 2012;344:e2124.

40. Amato MB, Meade MO, Slutsky AS, Brochard L, Costa EL, Schoenfeld DA, et al. Driving pressure and survival in the acute respiratory distress syndrome. N Engl J Med. 2015;372(8):747-55.

41. Pintado MC, de Pablo R, Trascasa M, Milicua JM, Rogero S, Daguerre M, et al. Individualized PEEP setting in subjects with ARDS: a randomized controlled pilot study. Respir Care. 2013;58(9):1416-23.

42. Aoyama H, Pettenuzzo T, Aoyama K, Pinto R, Englesakis M, Fan E. Association of driving pressure with mortality among ventilated patients with acute respiratory distress syndrome: a systematic review and metaanalysis. Crit Care Med. 2018;46(2):300-6.

43. Guervilly C, Forel JM, Papazian L. Respiratory rate and peak inspiratory pressure, new targets from the LUNG SAFE study analysis or physiopathological artifacts? J Thorac Dis. 2017;9(2):225-7.

44. Rich PB, Reickert CA, Sawada S, Awad SS, Lynch WR, Johnson KJ, et al. Effect of rate and inspiratory flow on ventilator-induced lung injury. J Trauma. 2000;49(5):903-11.

45. Protti A, Maraffi T, Milesi M, Votta E, Santini A, Pugni $P$, et al. Role of strain rate in the pathogenesis of ventilator-induced lung edema. Crit Care Med. 2016;44(9):e838-45.

46. Brander L, Slutsky AS. Invasive mechanical ventilation. In: Albert RK, Spiro SG, Jett JR, editors. Clinical respiratory medicine. 3rd ed. Philadelpia: Mosby/ Elsevier; 2008. p. 233

47. Bernard GR, Lucht WD, Niedermeyer ME, Snapper JR, Ogletree ML, Brigham $\mathrm{KL}$. Effect of $\mathrm{N}$-acetylcysteine on the pulmonary response to endotoxin in the awake sheep and upon in vitro granulocyte function. J Clin Invest. 1984; 73(6):1772-84

48. Snapper JR, Hutchison AA, Ogletree ML, Brigham KL. Effects of cyclooxygenase inhibitors on the alterations in lung mechanics caused by endotoxemia in the unanesthetized sheep. J Clin Invest. 1983;72(1):63-76.

49. Hutchison AA, Hinson JM Jr, Brigham KL, Snapper JR. Effect of endotoxin on airway responsiveness to aerosol histamine in sheep. J Appl Physiol Respir Environ Exerc Physiol. 1983;54(6):1463-8.

50. Dellacà RL, Zannin E, Sancini G, Rivolta I, Leone BE, Pedotti A, et al. Changes in the mechanical properties of the respiratory system during the development of interstitial lung edema. Respir Res. 2008;9(1):51.

51. Fuller BM, Mohr NM, Drewry AM, Carpenter CR. Lower tidal volume at initiation of mechanical ventilation may reduce progression to acute respiratory distress syndrome: a systematic review. Crit Care. 2013;17(1):R11.

52. Neto AS, Hemmes SN, Barbas CS, Beiderlinden M, Fernandez-Bustamante A,

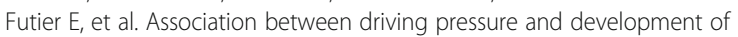
postoperative pulmonary complications in patients undergoing mechanical ventilation for general anaesthesia: a meta-analysis of individual patient data. Lancet Respir Med. 2016;4(4):272-80.

53. Loffreda S, Yang SQ, Lin HZ, Karp CL, Brengman ML, Wang DJ, et al. Leptin regulates proinflammatory immune responses. FASEB J. 1998;12(1):57-65.

54. Shah D, Romero F, Guo Z, Sun J, Li J, Kallen CB, et al. Obesity-induced endoplasmic reticulum stress causes lung endothelial dysfunction and promotes acute lung injury. Am J Respir Cell Mol Biol. 2017;57(2):204-15.

55. Bajwa EK, Yu CL, Gong MN, Thompson BT, Christiani DC. Pre-B-cell colonyenhancing factor gene polymorphisms and risk of acute respiratory distress syndrome. Crit Care Med. 2007;35(5):1290-5.

56. Gajic O, Dabbagh O, Park PK, Adesanya A, Chang SY, Hou P, et al. Early identification of patients at risk of acute lung injury: evaluation of lung injury prediction score in a multicenter cohort study. Am J Respir Crit Care Med. 2011;183(4):462-70.

\section{Publisher's Note}

Springer Nature remains neutral with regard to jurisdictional claims in published maps and institutional affiliations. 